\title{
BMJ Open Optimal cut points of plasma and urine neutrophil gelatinase-associated lipocalin for the prediction of acute kidney injury among critically ill adults: retrospective determination and clinical validation of a prospective multicentre study
}

Kristen M Tecson, ${ }^{1,2,3}$ Elisabeth Erhardtsen, ${ }^{4}$ Peter M Eriksen, ${ }^{4}$ A Osama Gaber, ${ }^{5}$ Michael Germain, ${ }^{6}$ Ladan Golestaneh, ${ }^{7}$ Maria de los Angeles Lavoria, ${ }^{4}$ Linda W Moore, ${ }^{5}$ Peter A McCullough ${ }^{1,3,8,9}$

To cite: Tecson KM, Erhardtsen E, Eriksen PM, et al. Optimal cut points of plasma and urine neutrophil gelatinase-associated lipocalin for the prediction of acute kidney injury among critically ill adults: retrospective determination and clinical validation of a prospective multicentre study. BMJ Open 2017;7:e016028. doi:10.1136/ bmjopen-2017-016028

- Prepublication history and additional material is available. To view please visit the journal (http://dx.doi.org/ 10.1136/ bmjopen-2017-016028).

Received 23 January 2017 Revised 9 May 2017 Accepted 9 May 2017

\section{CrossMark}

For numbered affiliations see end of article.

Correspondence to Dr Peter A McCullough; peteramccullough@gmail.com

\section{ABSTRACT}

Objectives To determine the optimal threshold of blood and urine neutrophil gelatinase-associated lipocalin (NGAL) to predict moderate to severe acute kidney injury (AKI) and persistent moderate to severe AKI lasting at least 48 consecutive hours, as defined by an adjudication panel. Methods A multicentre prospective observational study enrolled intensive care unit (ICU) patients and recorded daily ethylenediaminetetraacetic acid (EDTA) plasma, heparin plasma and urine NGAL. We used natural logtransformed NGAL in a logistic regression model to predict stage 2/3 AKI (defined by Kidney Disease International Global Organization). We performed the same analysis using the NGAL value at the start of persistent stage $2 / 3$ AKI.

Results Of 245 subjects, 33 (13.5\%) developed stage 2/3 AKI and 25 (10.2\%) developed persistent stage 2/3 AKI. Predicting stage $2 / 3$ AKI revealed the optimal NGAL cutoffs in EDTA plasma $(142.0 \mathrm{ng} / \mathrm{mL})$, heparin plasma $(148.3 \mathrm{ng} /$ $\mathrm{mL})$ and urine $(78.0 \mathrm{ng} / \mathrm{mL})$ and yielded the following decision statistics: sensitivity (SN) $=78.8 \%$, specificity $(\mathrm{SP})=73.0 \%$, positive predictive value $(\mathrm{PPV})=31.3 \%$, negative predictive value (NPV) $=95.7 \%$, diagnostic accuracy (DA) $=73.8 \%$ (EDTA plasma); $\mathrm{SN}=72.7 \%$, $\mathrm{SP}=73.8 \%, \mathrm{PPV}=30.4 \%, \mathrm{NPV}=94.5 \%, \mathrm{DA}=73.7 \%$ (heparin plasma); $\mathrm{SN}=69.7 \%, \mathrm{SP}=76.8 \%, \mathrm{PPV}=32.9 \%, \mathrm{NPV}=94 \%$, $\mathrm{DA}=75.8 \%$ (urine). The optimal NGAL cutoffs to predict persistent stage $2 / 3 \mathrm{AKI}$ were similar: $148.3 \mathrm{ng} / \mathrm{mL}$ (EDTA plasma), $169.6 \mathrm{ng} / \mathrm{mL}$ (heparin plasma) and $79.0 \mathrm{ng} / \mathrm{mL}$ (urine) yielding: $\mathrm{SN}=84.0 \%, \mathrm{SP}=73.5 \%, \mathrm{PPV}=26.6 \%$, $\mathrm{NPV}=97.6, \mathrm{DA}=74.6 \%$ (EDTA plasma), $\mathrm{SN}=84 \%$, $\mathrm{SP}=76.1 \%, \mathrm{PPV}=26.8 \%, \mathrm{NPV}=96.5 \%, \mathrm{DA}=76.1 \%$ (heparin plasma) and $\mathrm{SN}=75 \%, \mathrm{SP}=75.8 \%, \mathrm{PPV}=26.1$, $\mathrm{NPV}=96.4 \%, \mathrm{DA}=75.7 \%$ (urine).

Conclusion Blood and urine NGAL predicted stage $2 / 3 \mathrm{AKI}$, as well as persistent $2 / 3 \mathrm{AKI}$ in the ICU with acceptable decision statistics using a single cut point in each type of specimen.
Strengths and limitations of this study

- This study used biomarker data from both plasma and urine samples.

- Acute kidney injury diagnosis was adjudicated by an expert panel.

- We used an unbiased, data-driven approach to identify a single cut point for each plasma and urinary NGAL sample to predict stage 2 or 3 acute kidney injury and persistent stage 2 or 3 acute kidney injury.

- This small prospective cohort study had limited clinical follow-up.

\section{INTRODUCTION}

Serum creatinine and urine output are used to diagnose acute kidney injury (AKI) as defined by the 2012 Kidney Disease International Global Organization (KDIGO) guidelines (see online supplementary table 1); however, creatinine is an imperfect marker as it peaks anywhere from 2 to 7 days following an insult to the kidneys. ${ }^{1}$ Additionally, urine output may lead to an inaccurate diagnosis of AKI in the intensive care unit (ICU), as it is commonly manipulated in the ICU through use of intravenous fluids and diuretics. Neutrophil gelatinase-associated lipocalin (NGAL) has been shown to peak following tubular injury earlier than changes in creatinine and urine output, making it a desirable biomarker for predicting the development of AKI. ${ }^{2}$ Previous studies have shown the utility of this biomarker, but have suffered from heterogeneous AKI thresholds and 
lack of validation of parenchymal renal involvement in the injury. In those studies, AKI may have been due to a transient haemodynamically related reduction in renal filtration due to poor forward perfusion or impaired plasma refill, which represents a distinct AKI pathophysiology. ${ }^{3}$ Furthermore, the optimal body fluid for NGAL assay performance (blood or urine) is unknown, as is the optimal cut point for the detection of moderate to severe AKI (KDIGO stage 2 or 3). We set out to use a commercially available NGAL assay in a multicentre, blinded study to assess its performance in two types of plasma samples and in one urine sample for the prediction of moderate to severe AKI, as defined by KDIGO and adjudicated by clinicians blinded to the NGAL values.

\section{METHODS}

\section{Study details}

This study prospectively enrolled consecutive adult patients admitted to an ICU or critical care setting after obtaining informed consent between 5 March 2014 and 15 April 2015 from four participating hospitals in Boston and Springfield, Massachusetts; Bronx, New York; and Houston, Texas; the protocol may be found at the following: http://www.bioporto.com/Products/ Featured-areas/NGAL.aspx. Each study site received institutional review board (IRB) approval prior to enrolling patients (IRBs used for this study are as follows: Baystate Health IRB \#1; Houston Methodist Research Institute
IRB 1; Partners Human Research Committee; Biomedical Research Alliance of New York IRB). Those with history of nephrectomy, renal transplantation and/or renal replacement therapy initiated before admission were not eligible to participate. In addition to the standard clinical care and laboratory testing, one urine and two plasma samples were drawn and frozen per day in the ICU, up to 8 days. The samples were shipped to a central laboratory where NGAL was assayed by BioPorto Diagnostics A/S, Copenhagen, Denmark. This test has a measurable range of $25-3000 \mathrm{ng} / \mathrm{mL} .^{5}$ At mean NGAL concentrations of 97, 116 and $112 \mathrm{ng} / \mathrm{mL}$ in EDTA plasma, heparin plasma and urine, respectively, the coefficients of variation were $3.1 \%, 1.8 \%$ and $2.8 \%$, respectively. If patients were discharged early from the ICU during the study period, serum creatinine levels were collected manually from the hospital data system for 48 hours provided the patient was still hospitalised. Reference standard AKI was determined daily, up to 8 days, by a three-physician adjudication panel using the KDIGO guidelines. AKI was confirmed by the adjudication panel, and disagreements were settled by conference calls and discussion of the cases. Adjudicators were blinded to the NGAL values and physician notes concerning the renal diagnosis. ${ }^{6}$

\section{Patient involvement}

Patient involvement began at the time of informed consent; patients and caregivers were not involved in the
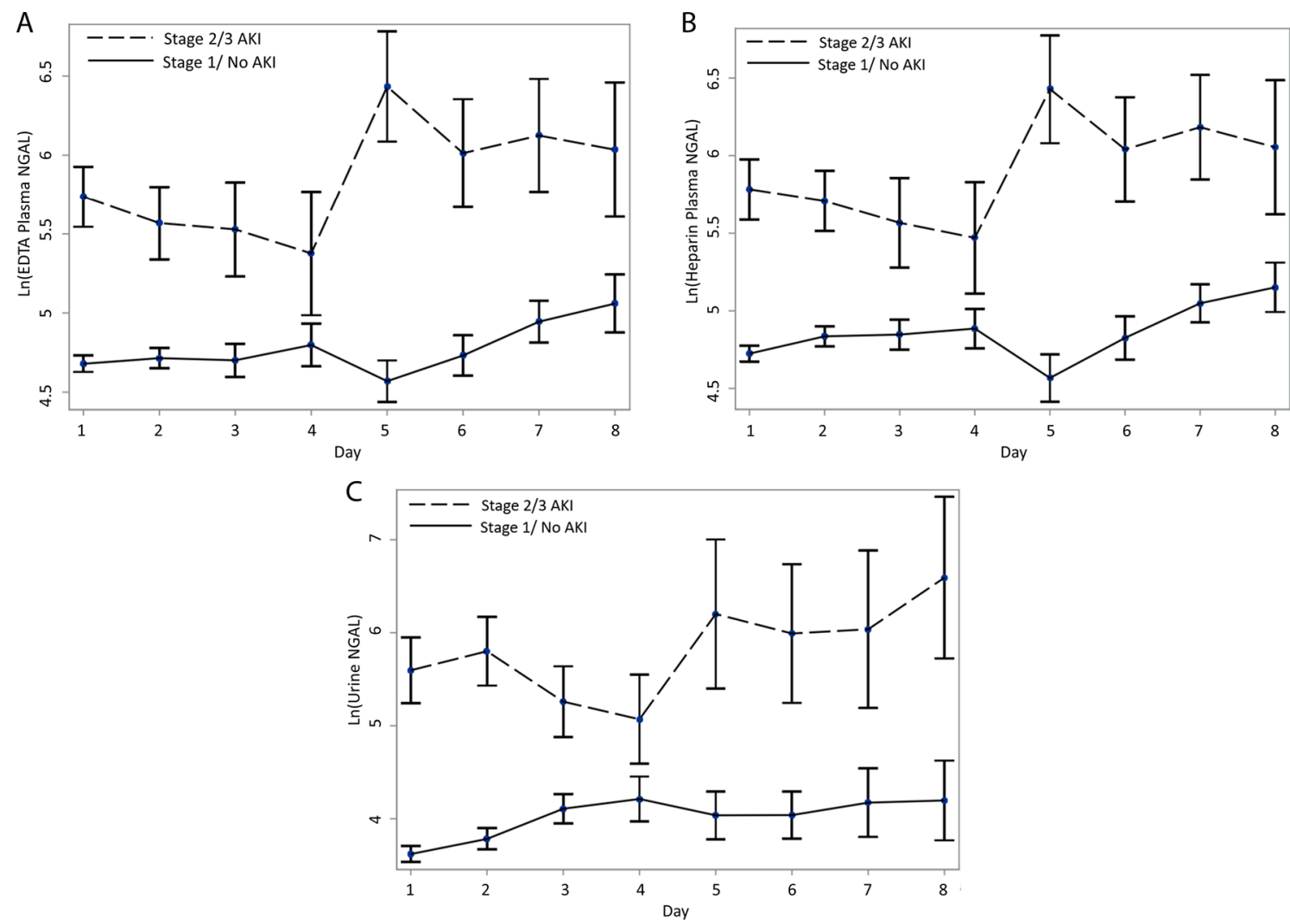

Figure 1 Mean and standard error of natural log-transformed NGAL by day and acute kidney injury status ((A) EDTA plasma; (B) heparin plasma; (C) urine). AKI, acute kidney injury; EDTA, ethylenediaminetetraacetic acid; NGAL, neutrophil gelatinaseassociated lipocalin. 
design of, recruitment for, or conduct of this study. The development of the outcome measures was not informed by patients' priorities, experiences or preferences. The participants' contributions to this study are deeply appreciated, and the results of the study will be publicly available to the patients via this publication.

\section{Analyses}

This manuscript was constructed via retrospective analyses of prospectively collected data and was developed under STAndards for the Reporting of Diagnostic accuracy studies guidelines; ethics committee approval was obtained prior to conducting these analyses. NGAL values were highly skewed in all three sample types so we used the natural-log transformed distributions (figure $1 \mathrm{~A}-\mathrm{C}$ ). We performed logistic regression using the natural log-transformed baseline NGAL (i.e. $\ln$ (baseline NGAL)) value as a predictor for the outcome of moderate/severe (stage 2/3) AKI at any point during observation. Additionally, we defined persistent moderate/severe (stage 2/3) AKI as two or more days of consecutive stage $2 / 3$ AKI, and we constructed another logistic regression model for the outcome of persistent 2/3 AKI using a wandering $\ln$ (baseline NGAL) value as the predictor variable. The wandering $\ln$ (baseline NGAL) value was taken on the morning of the first day of a 48-hour persistent AKI period. If AKI did not occur, the wandering $\ln$ (baseline NGAL) value was taken to be the $\ln$ (baseline NGAL). We found the optimal NGAL value for both outcomes by using the criterion of smallest distance to the 'perfect point' on the receiver operating characteristic (ROC) curve, $(0,1)$. Baseline NGAL values $<5 \mathrm{ng} / \mathrm{mL}$ were adjusted using a regression model, which affected approximately $2 \%$ of the data. We assessed differences in baseline characteristics between those who had at least 1 day of stage 2/3 AKI at any time

Table 1 Patient characteristics by occurrence of moderate/severe acute kidney injury (AKI)

\begin{tabular}{|c|c|c|c|}
\hline Variable & Stage 2 or 3 AKI $(n=33)$ & Stage 1 or no AKI (n=212) & p Value \\
\hline Age (years) & $68(56,74)$ & $63(54,73)$ & 0.50 \\
\hline Gender (male) (n (\%)) & $22(66.7)$ & $135(63.7)$ & 0.74 \\
\hline Race/ethnicity (n (\%)) & & & 0.86 \\
\hline African American & $3(9.1)$ & $29(13.7)$ & \\
\hline Asian & $0(0.0)$ & $2(0.9)$ & \\
\hline Hispanic or Latino & $4(12.1)$ & $28(13.2)$ & \\
\hline Mixed & $0(0.0)$ & $2(0.9)$ & \\
\hline White & $26(78.8)$ & $151(71.2)$ & \\
\hline Hypertension (n (\%)) & $26(78.8)$ & $142(67.0)$ & 0.17 \\
\hline Congestive heart failure (n (\%)) & $6(18.2)$ & $38(17.9)$ & 0.97 \\
\hline Diabetes mellitus ( $\mathrm{n}(\%)$ ) & & & 0.04 \\
\hline Type I & $0(0.0)$ & $2(0.9)$ & \\
\hline Type II & $16(48.5)$ & $57(26.9)$ & \\
\hline None & $17(51.5)$ & $153(72.2)$ & \\
\hline Urinary tract infection $(\mathrm{n}(\%))$ & $2(6.1)$ & $6(2.8)$ & 0.33 \\
\hline Sepsis (n (\%)) & $6(18.2)$ & $12(5.7)$ & 0.01 \\
\hline Chronic kidney disease history ( $\mathrm{n}(\%))$ & & & $<0.001$ \\
\hline None & $22(66.7)$ & $190(89.6)$ & \\
\hline Stage 1 & $1(3.0)$ & $4(1.9)$ & \\
\hline Stage 2 & $1(3.0)$ & $3(1.4)$ & \\
\hline Stage $3 A$ & $8(24.2)$ & $5(2.4)$ & \\
\hline Stage 3B & $0(0.0)$ & $8(3.8)$ & \\
\hline Stage 4 & $1(3.0)$ & $2(0.9)$ & \\
\hline In-hospital renal replacement therapy (n (\%)) & $4(12.1)$ & $2(0.9)$ & 0.003 \\
\hline Nephrotoxin use at baseline (n (\%)) & $18(54.6)$ & $88(41.5)$ & 0.19 \\
\hline Baseline EDTA NGAL (ng/mL) & $276(155,517)$ & $98(70,154)$ & $<0.001$ \\
\hline Baseline heparin plasma NGAL $(\mathrm{ng} / \mathrm{mL})$ & $288(150,669)$ & $100(68,160)$ & $<0.001$ \\
\hline Baseline urine NGAL (ng/mL) & $190(61,1133)$ & $31(15,67)$ & $<0.001$ \\
\hline Days in intensive care unit & $4(2,8)$ & $2(1,3)$ & 0.001 \\
\hline
\end{tabular}

EDTA, ethylenediaminetetraacetic acid; NGAL, neutrophil gelatinase-associated lipocalin. 
during observation and those who did not via Wilcoxon rank sum and $\chi^{2}$ tests; we did the same for those who had persistent stage $2 / 3$ AKI and those who did not have persistent stage $2 / 3 \mathrm{AKI}$ at any point during observation. Continuous variables are reported as medians (quarter 1, quarter 3). Categorical variables are reported as frequencies and proportions.

\section{RESULTS}

A total of 252 patients were screened for the study; two were screen failures, and five did not have sufficient information to be included in analyses. Hence, 245 patients were enrolled and included in analyses. Only two patients in this study had cardiac surgery during the observational period. No adverse events occurred during the observation period. There were $33(13.5 \%)$ subjects who developed stage 2/3 AKI as determined by the adjudicators. With this event rate, we had $95 \%$ power to detect an area under the curve (AUC) as low as 0.69. Age and gender did not differ significantly between those with or without stage 2/3 AKI; however, those with stage 2/3 AKI had more comorbidities (sepsis: $18.2 \%$ vs $5.7 \%, \mathrm{p}=0.01$; diabetes $48.5 \%$ vs $27.8 \%, \mathrm{p}=0.04$; chronic kidney disease $(\mathrm{CKD})$ : $33.3 \%$ vs $10.4 \%, \mathrm{p}<0.001)$, longer ICU stays $(4(2,8)$ days vs $2(1,3)$ days, $\mathrm{p}=0.001)$ and higher baseline NGAL levels (EDTA plasma: $276(155,517) \mathrm{ng} / \mathrm{mL}$ vs $98(70,154) \mathrm{ng} /$ $\mathrm{mL}, \mathrm{p}<0.001$; heparin plasma: $288(150,669) \mathrm{ng} / \mathrm{mL}$ vs $100(68,160) \mathrm{ng} / \mathrm{mL}, \mathrm{p}<0.001$; urine: 190 (61, 1133) $\mathrm{ng} / \mathrm{mL}$ vs $31(15,67), \mathrm{p}<0.001)$ than those without AKI (table 1). Using the $\ln$ (baseline EDTA plasma NGAL) as the independent variable in a logistic regression model for the outcome of stage 2/3 AKI at any time yielded an $\mathrm{AUC}=0.76,95 \%$ CI 0.64 to $0.87, \mathrm{p}<0.0001$ (figure $2 \mathrm{~A}$ ). Optimising the ROC curve with respect to the distance from $(0,1)$ resulted in a cut point of $142.0 \mathrm{ng} / \mathrm{mL}$, which yielded a sensitivity (SN) of $78.8 \%$, specificity (SP) of $73.0 \%$, positive predictive value (PPV) of $31.3 \%$, negative predictive value (NPV) of $95.7 \%$ and diagnostic accuracy (DA) of $73.8 \%$. Similar results are shown for heparin plasma and urine NGAL in figure $2 \mathrm{~B}$ and $\mathrm{C}$.

Twenty-five (10.2\%) subjects had persistent stage 2/3 AKI. Demographics did not differ significantly between those who did and those who did not have persistent stage 2/3 AKI; however, those with persistent stage 2/3 AKI had a higher rate of CKD (36.0\% vs $10.9 \%, \mathrm{p}<0.001)$, longer ICU stays $(6(2,8)$ days vs $2(1,3)$ days, $\mathrm{p}<0.001)$ and higher wandering baseline NGAL values (EDTA plasma: $287(188,517) \mathrm{ng} / \mathrm{mL}$ vs $98(70,156) \mathrm{ng} / \mathrm{mL}, \mathrm{p}<0.001$; heparin: $355(178,716) \mathrm{ng} / \mathrm{mL}$ vs $101(68,162) \mathrm{ng} /$ $\mathrm{mL}, \mathrm{p}<0.001$; urine: $231.1(84.3,1203) \mathrm{ng} / \mathrm{mL}$ vs 32 (15, 75) $\mathrm{ng} / \mathrm{mL}, \mathrm{p}<0.001$ ) (table 2). We used the wandering $\ln$ (baseline EDTA plasma NGAL) in a logistic regression model to predict persistent stage $2 / 3 \mathrm{AKI}$, which yielded an $\mathrm{AUC}=0.85,95 \%$ CI 0.77 to $0.94, \mathrm{p}<0.001$. The optimal cut point for NGAL based on the distance to $(0,1)$ was $148.3 \mathrm{ng} / \mathrm{mL}$ (figure $3 \mathrm{~A}$ ). This value yielded $\mathrm{SN}=84.0 \%$, $\mathrm{SP}=73.5 \%, \mathrm{PPV}=26.6 \%, \mathrm{NPV}=97.6 \%, \mathrm{DA}=74.6 \%$. Similar cut points and results are shown for heparin plasma and
A

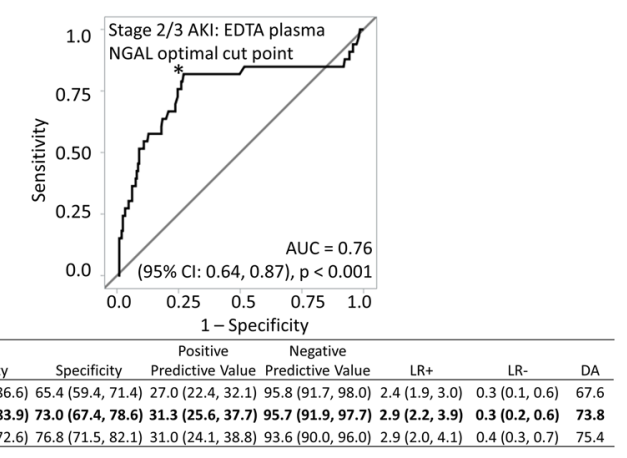

B

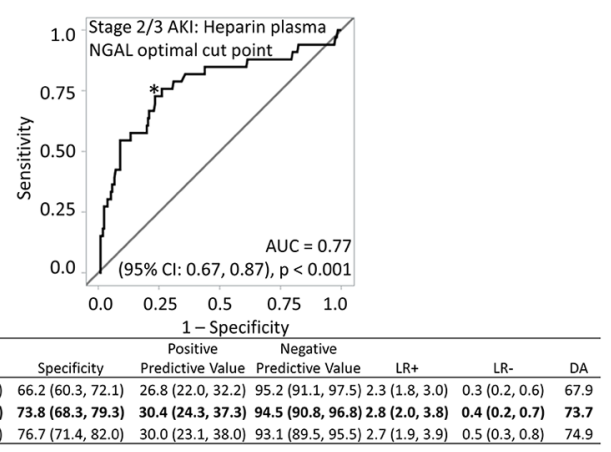

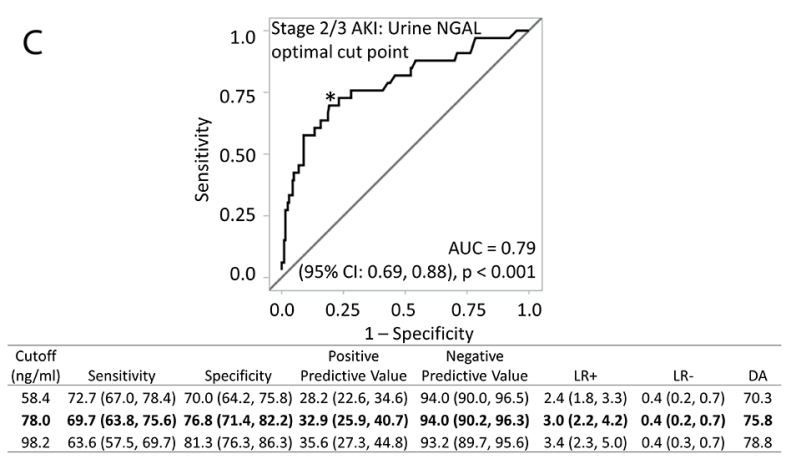

Figure 2 Receiver operating characteristic curves for natural log-transformed baseline NGAL as a predictor of stage 2/3 acute kidney injury with the optimal NGAL threshold identified ((A) EDTA plasma; (B) heparin plasma; (C) urine). Decision statistics are presented for the optimal cut point (bold) as well as at cut points approximately $20 \mathrm{ng} / \mathrm{mL}$ below and above the optimal value. $L R+$ indicates positive likelihood ratio, LR- indicates negative likelihood ratio, and DA indicates diagnostic accuracy. AUC, area under the curve; EDTA, ethylenediaminetetraacetic acid; NGAL, neutrophil gelatinase-associated lipocalin. 
urine NGAL in figure $3 \mathrm{~B}$ and $\mathrm{C}$. Odds ratios from the regression models are shown in table 3 .

\section{DISCUSSION}

We found that a single measured threshold for NGAL, either in blood or urine, was effective in risk prediction for the development of moderate to severe AKI and persistent AKI, as defined by AKI lasting 48 consecutive hours or longer. NGAL measured on a daily basis performed well in the prognostication of persistent AKI. Our results were internally consistent and add to the literature as they yielded unbiased cut points from prospectively collected samples and adjudicated AKI outcomes. We found that urine and plasma yielded different absolute NGAL concentrations; however, within each sample type the cut point for the prediction of stage 2/3 AKI or persistent AKI was similar, making daily measurements and utilisation of a single threshold feasible, provided there is consistency in sample source and handling procedures. Our results are concordant with a prior study that observed slightly better discriminatory power of plasma NGAL than urinary NGAL, with an AUC as high as $0.84 .{ }^{7}$ Thus, we believe, for ease of use, plasma NGAL is more likely to be incorporated into routine care than urinary NGAL.

Our findings build on the work of others using a variety of NGAL assays demonstrating the concept that 25 kilodalton protein is rapidly and reliably upregulated after ischaemia/reperfusion injury to the kidney. ${ }^{89}$ The work by Nickolas et al revealed that NGAL even has the ability to distinguish AKI from non-progressive CKD, a feat that creatinine has failed to accomplish. ${ }^{10}$ Furthermore, the urine NGAL measured in the emergency department was associated with the development of end-stage renal disease requiring dialysis in the hospital. A 2010-2011

Table 2 Sample characteristics for subjects with and without persistent stage 2/3 AKI

\begin{tabular}{|c|c|c|c|}
\hline Variable & Persistent AKI ( $n=25)$ & No persistent AKI $(n=220)$ & p Value \\
\hline Age (years) & $68(60,73)$ & $63(54,73)$ & 0.31 \\
\hline Gender (male) (n (\%)) & $16(64.0)$ & $141(64.1)$ & 0.99 \\
\hline Race/ethnicity (n (\%)) & & & 0.89 \\
\hline African American & $2(8.0)$ & $30(13.6)$ & \\
\hline Asian & $0(0.0)$ & $2(0.9)$ & \\
\hline Hispanic or Latino & $3(12.0)$ & $29(13.2)$ & \\
\hline Mixed & $0(0.0)$ & $2(0.9)$ & \\
\hline White & $20(80.0)$ & $157(71.4)$ & \\
\hline Hypertension (n (\%)) & $19(76.0)$ & $149(67.7)$ & 0.40 \\
\hline Congestive heart failure (n (\%)) & $4(16.0)$ & $40(18.2)$ & 0.79 \\
\hline Diabetes mellitus (n (\%)) & & & 0.40 \\
\hline Type I & $0(0.0)$ & $2(0.9)$ & \\
\hline Type II & $10(40.0)$ & $63(28.6)$ & \\
\hline None & $15(60.0)$ & $155(70.5)$ & \\
\hline Urinary tract infection (n (\%)) & $2(8.0)$ & $6(2.73)$ & 0.19 \\
\hline Sepsis (n (\%)) & $4(16.0)$ & $14(6.4)$ & 0.10 \\
\hline Chronic kidney disease history ( $\mathrm{n}(\%))$ & & & $<0.001$ \\
\hline None & $16(64.0)$ & $196(89.1)$ & \\
\hline Stage 1 & $1(4.0)$ & $4(1.8)$ & \\
\hline Stage 2 & $1(4.0)$ & $3(1.4)$ & \\
\hline Stage $3 \mathrm{~A}$ & $6(24.0)$ & $7(3.2)$ & \\
\hline Stage 3B & $0(00)$ & $8(3.6)$ & \\
\hline Stage 4 & $1(94.0)$ & $2(0.9)$ & \\
\hline In-hospital renal replacement therapy (n (\%)) & $4(16.0)$ & $2(0.9)$ & 0.001 \\
\hline Use of nephrotoxins ( $(\%))$ & $14(56.05)$ & $92(41.8)$ & 0.18 \\
\hline Wandering baseline EDTA NGAL $(\mathrm{ng} / \mathrm{mL})$ & $287(188,517)$ & $98(70,156)$ & $<0.001$ \\
\hline Wandering baseline heparin plasma NGAL $(\mathrm{ng} / \mathrm{mL})$ & $355(178,716)$ & $101(68,162)$ & $<0.001$ \\
\hline Wandering baseline urine NGAL $(\mathrm{ng} / \mathrm{mL})$ & $231.1(84.3,1203)$ & $32(15,75)$ & $<0.001$ \\
\hline Days in intensive care unit & $6(2,8)$ & $2(1,3)$ & $<0.001$ \\
\hline
\end{tabular}

AKI, acute kidney injury; EDTA, ethylenediaminetetraacetic acid; NGAL, neutrophil gelatinase-associated lipocalin. 
A

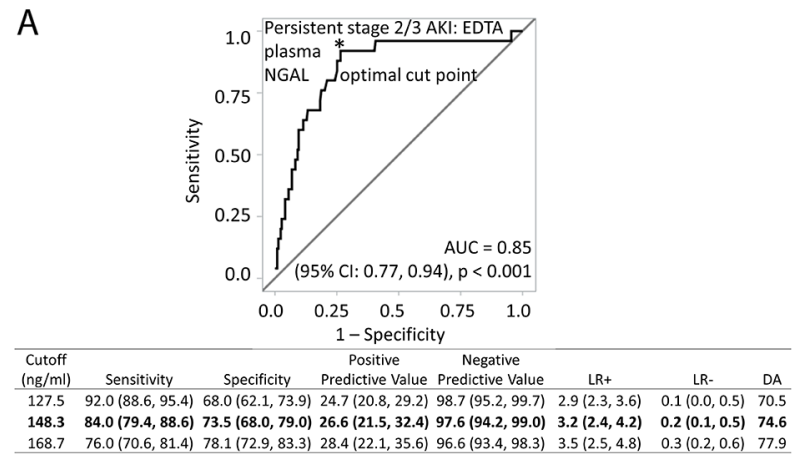
B

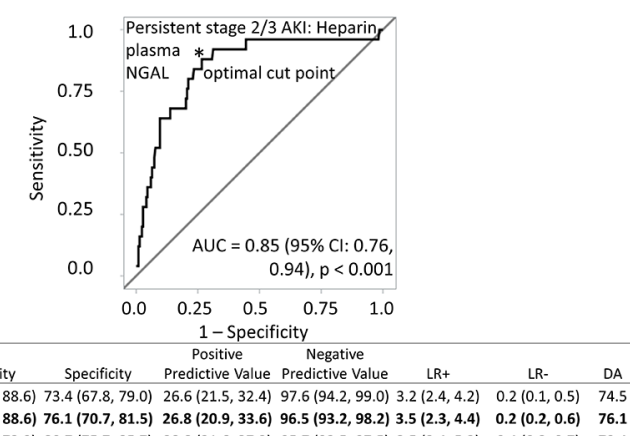

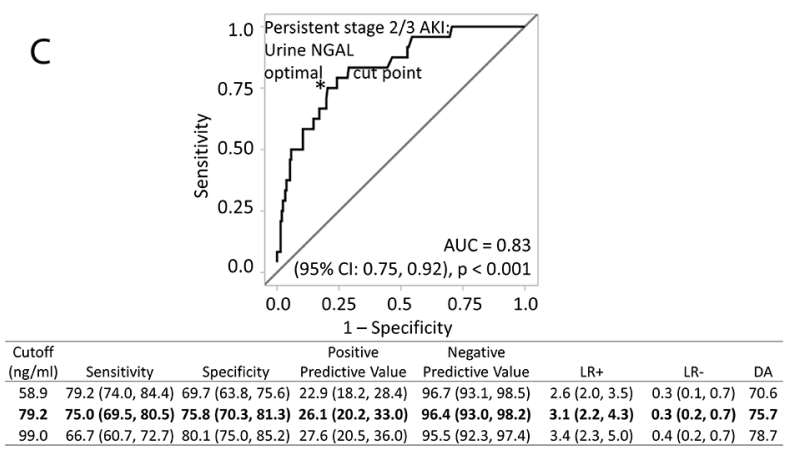

Figure 3 Receiver operating characteristic curves for wandering natural log-transformed baseline NGAL as a predictor of persistent stage 2/3 acute kidney injury with the optimal NGAL threshold identified ((A) EDTA plasma; (B) heparin plasma; (C) urine). Decision statistics are presented for the optimal cut point (bold) as well as at cut points approximately $20 \mathrm{ng} / \mathrm{mL}$ below and above the optimal value. LR+ indicates positive likelihood ratio,LR- indicates negative likelihood ratio, and DA indicates diagnostic accuracy. AUC, area under the curve; EDTA, ethylenediaminetetraacetic acid; NGAL, neutrophil gelatinaseassociated lipocalin.

study in Western Australia, $n=102$, demonstrated the effectiveness of using NGAL to predict inpatient AKI (based on risk, injury, failure, loss of kidney function and end-stage kidney disease classification) in a tertiary care setting. In the Australian study, the Biosite Triage NGAL in whole blood (Alere, Inverness Medical, Australia) was found to have a single optimal cut-off of $89 \mathrm{ng} / \mathrm{mL}$ and had $\mathrm{SN}=68 \%, \mathrm{SP}=70 \%$ and $\mathrm{AUC}=0.71,95 \% \mathrm{CI} 0.58$ to $0.84 .{ }^{11}$ While the threshold was similar to that found in our EDTA and heparin samples, this study used all stages of AKI as the endpoint and did not have blinded adjudication by a committee. Nevertheless, these data are in line with our conclusion that NGAL measured in blood performs well as a prognostic aid for AKI. A meta-analysis of 1478 patients with sepsis demonstrated that NGAL was associated with the development of AKI, need for renal replacement therapy and mortality. ${ }^{12}$ Marino $e t a l \mathrm{~s}$ study of 101 emergency department patients with sepsis confirmed that NGAL was related to (the severity of) AKI; however, NGAL concentrations were also elevated in the absence of AKI in these patients. ${ }^{13}$ Numerous studies have demonstrated that NGAL rises after cardiac surgery and is congruent with AKI. ${ }^{1415}$ Additionally, NGAL concentrations increase after contrast-induced AKI; however, it is to a much lesser extent than from cardiac surgery or critical illness. ${ }^{16}$ An extensive literature review and meta-analysis by Haase $e t$ al revealed a wide range of optimal reported NGAL cutoffs for AKI prediction $(100-270 \mathrm{ng} / \mathrm{mL}){ }^{17}$ The optimal plasma cutoffs found in our study to detect stage 2/3 AKI (EDTA: $142.0 \mathrm{ng} / \mathrm{mL}$, heparin: $149.5 \mathrm{ng} /$

Table 3 ORs for stage 2/3 acute kidney injury and persistent stage 2/3 acute kidney injury using EDTA, heparin and urine NGAL values as predictors (odds are associated with an approximate 2.73-fold increase in NGAL value)

\begin{tabular}{llll}
\hline Outcome & NGAL measure & p Value & OR (95\% Cl) \\
\hline Stage 2/3 acute kidney injury & EDTA & $<0.001$ & $2.8(1.8$ to 4.4$)$ \\
& Heparin & $<0.001$ & $3.2(2.0$ to 4.9$)$ \\
Persistent stage 2/3 acute kidney injury & EDTA & $<0.001$ & $2.2(1.7$ to 2.9$)$ \\
& Heparin & $<0.001$ & $3.8(2.3$ to 6.3$)$ \\
& Urine & $<0.001$ & $3.9(2.3$ to 6.3$)$ \\
\hline
\end{tabular}

EDTA, ethylenediaminetetraacetic acid; NGAL, neutrophil gelatinase-associated lipocalin. 
$\mathrm{mL}$ ) were within the range reported in the meta-analysis. Haase et al suggested cutoffs between $150 \mathrm{ng} / \mathrm{mL}$ and $170 \mathrm{ng} / \mathrm{mL}$ for adults, which is slightly higher than our findings and may be attributable to the high rate of postoperative patients used in the meta-analysis, as well as the varied definitions of AKI. Our study is the first among these to demonstrate that NGAL can be used to risk predict stage $2 / 3 \mathrm{AKI}$ and its persistence in a cohort of ICU patients. We further demonstrated that a single cut-off value performs well in terms of decision statistics for the outcomes of stage $2 / 3 \mathrm{AKI}$ and persistent stage 2/3 AKI.

There have been large prospective studies examining novel biomarkers other than NGAL for the prediction of stage 2/3 AKI among the critically ill. Kashani and colleagues $(n=728)$ measured the concentrations of urinary tissue inhibitor of metalloproteinases (TIMP)-2 and insulin-like growth factor binding protein 7 (IGFBP7), and combined the two measures into one predictor, referred to as (TIMP-2)*(IGFBP7), NephroCheck (Astute Medical, San Diego, California, USA) to identify imminent stage $2 / 3$ AKI (ie, in the next 12 hours), which occurred in $14 \%$ of study participants. Baseline urinary (TIMP-2)*(IGFBP7) yielded an $\mathrm{AUC}=0.80,97 \% \mathrm{CI} 0.76$ to 0.83 ; however, the full decision statistics of an optimal cut point were not disclosed. ${ }^{18} \mathrm{~A}$ second study by Bihorac and colleagues $(n=420)$ evaluated urinary (TIMP-2)*(IGFBP7) for the same endpoint. In this study, $71(17.4 \%)$ patients had imminent stage 2/3 AKI and the model had an AUC $=0.82,95 \%$ CI 0.76 to 0.88 . However, (TIMP-2)*(IGFBP7) did not appear amenable to a single threshold value. Two cut points were presented: one yielding high SN and low SP and the other yielding low SN and high SP. At the cut-off of $0.3(\mathrm{ng} / \mathrm{mL})^{2} / 1000$, the $\mathrm{SN}=92,95 \%$ CI $85 \%$ to $98 \%$, but the $\mathrm{SP}=46,95 \%$ CI $41 \%$ to $52 \%$. At a sevenfold higher cut point of $2.0(\mathrm{ng} / \mathrm{mL})^{2} / 1000$, the $\mathrm{SN}=37,95 \%$ CI $26 \%$ to $47 \%$ and $\mathrm{SP}=95,95 \%$ CI $93 \%$ to $97 \% .{ }^{19}$ In comparison, an NGAL EDTA plasma level of $142.0 \mathrm{ng} / \mathrm{mL}$ in our study predicted stage 2/3 AKI and yielded $\mathrm{SN}=78.8,95 \% \mathrm{CI}$ $73.7 \%$ to $83.9 \%$ and $\mathrm{SP}=73.0,95 \%$ CI $67.4 \%$ to $78.6 \%$. Thus, for NGAL at a single cut point, the lower bounds of the $95 \%$ CIs for sensitivity and specificity were well above $60 \%$ and $40 \%$, respectively. Accordingly, NGAL appears to have considerable advantages over (TIMP-2)*(IGFBP7) in forecasting AKI over a longer time window, at a single prognostic threshold, and avoids low sensitivity or specificity. Additionally, we demonstrated NGAL performed well when measured daily using similar cut points for the prediction of persistent stage 2/3 AKI. Daily use in risk prediction has not been demonstrated in any study with (TIMP2)*(IGFBP7).

\section{LIMITATIONS}

Our study has all the limitations of small prospective cohort studies of biomarkers including limited clinical follow-up and lack of precise follow-up information, including accurate urine output. We did not consider stage I AKI in the definition of the primary endpoint because prior studies suggested that many in this category could have transient haemodynamic elevations of creatinine without parenchymal AKI. Thus, our event rate is lower than other studies that included all KDIGO stages of AKI. Our study sample was non-homogeneous in that it included patients with sepsis, a diagnosis that has been shown to increase NGAL levels even for those without AKI. ${ }^{13}$ Furthermore, due to the small sample size, we could not analyse sepsis or CKD subjects separately, rendering it impossible to determine whether different cutoffs were needed. To examine confounders' relations with (persistent) stage 2/3 AKI, we built multivariate models; however, neither sepsis nor CKD and DM were statistically significant when considered with NGAL simultaneously. Consequently, the adjusted OR estimates changed only slightly, and the corresponding $\mathrm{p}$ values were unchanged. Perhaps this is due, in part, to the fact that NGAL can distinguish AKI from non-progressive CKD. Additionally, the small sample size did not permit the use of training and/or validation sets, which prohibited further investigation of the models. However, all AUCs were at least 0.76 , which indicates good predictive ability. Finally, we did not have information on long-term outcomes such as need for renal replacement therapy or mortality, which would have been useful in integrating NGAL with serum creatinine and urine output in an in-depth assessment of patient outcomes. ${ }^{20}$

\section{CONCLUSION}

NGAL concentrations of $142.0,149.5$ and $78.0 \mathrm{ng} / \mathrm{mL}$ in EDTA plasma, heparin plasma and urine, respectively, were found to be optimal in predicting stage 2/3 AKI in ICU patients participating in a prospective observational study. Similarly, NGAL concentration thresholds of 148.3, 169.6 and $79.2 \mathrm{ng} / \mathrm{mL}$ in EDTA plasma, heparin plasma and urine, respectively, were optimal in predicting persistent stage 2/3 AKI in the same cohort of ICU patients. A larger prospective study to provide external validation of these cut points is warranted.

\section{Author affiliations}

${ }^{1}$ Baylor Heart and Vascular Institute, Dallas, Texas, USA

${ }^{2}$ Baylor Scott \& White Research Institute, Dallas, Texas, USA

${ }^{3}$ Texas A\&M College of Medicine Health Science Center, Dallas, Texas, USA

${ }^{4}$ BiPorto Diagnostics A/S, Copenhagen, Denmark

${ }^{5}$ Houston Methodist Hospital, Houston, Texas, USA

${ }^{6}$ Baystate Medical Center, Springfield, Massachusetts, USA

${ }^{7}$ Albert Einstein College of Medicine, Bronx, New York, USA

${ }^{8}$ Baylor University Medical Center, Dallas, Texas, USA

${ }^{9}$ Baylor Jack and Jane Hamilton Heart and Vascular Hospital, Dallas, Texas, USA

Acknowledgements The authors would like to thank the study participants who graciously shared their time and materials for the purposes of this research.

Contributors PAM conceived the study. PME funded the study. AOG, MG, LG and LWM initiated the study design and helped with implementation. KMT conducted the statistical analysis. KMT and PAM drafted the article. All authors contributed to the manuscript's refinement and approved the final version.

Funding The study was funded by BioPorto Diagnostics A/S. 
Competing interests EE, PME, and ML are employees of BioPorto Diagnostics A/S. The remaining authors have nothing to disclose.

Patient consent Obtained.

Ethics approval Institutional Review Board (Baystate Health IRB \#1; Houston Methodist Research Institute (HMRI) IRB 1; Pratners Human Research Committee; Biomedical Research Alliance of New York (BRANY) IRB).

Provenance and peer review Not commissioned; externally peer reviewed.

Data sharing statement The data from this study will not be made available.

Open Access This is an Open Access article distributed in accordance with the Creative Commons Attribution Non Commercial (CC BY-NC 4.0) license, which permits others to distribute, remix, adapt, build upon this work non-commercially, and license their derivative works on different terms, provided the original work is properly cited and the use is non-commercial. See: http://creativecommons.org/ licenses/by-nc/4.0/

(c) Article author(s) (or their employer(s) unless otherwise stated in the text of the article) 2017. All rights reserved. No commercial use is permitted unless otherwise expressly granted.

\section{REFERENCES}

1. KDIGO Clinical Practice Guideline for Acute kidney Injury. Kidney Int 2012;2. (suppl 1).

2. McCullough PA, Shaw AD, Haase M, et al. Diagnosis of acute kidney injury using functional and injury biomarkers: workgroup statements from the tenth acute dialysis quality initiative consensus conference. Contrib Nephrol 2013;182:13-29.

3. Chang $\mathrm{CH}$, Yang $\mathrm{CH}$, Yang $\mathrm{HY}$, et al. Urinary biomarkers improve the diagnosis of intrinsic acute kidney injury in coronary care units. Medicine 2015;94:e1703.

4. $\mathrm{Xu} \mathrm{K}$, Rosenstiel P, Paragas $\mathrm{N}$, et al. Unique transcriptional programs identify subtypes of AKI. J Am Soc Nephrol 2017.28.

5. The NGAL test ${ }^{\mathrm{TM}}$. Gentofte, Denmark: BioPorto Diagnostics A/S, 2013.

6. U.S Department of Health and Human Services, Food and Drug Administration, Center for Devices and Radiological Health. Guidance for Industry and FDA Staff: Statistical Guidance on Reporting Results from Studies Evaluating Diagnostic Tests. Washington, DC. Author. (13 Mar 2007).

7. Schley G, Köberle C, Manuilova E, et al. Comparison of plasma and urine biomarker performance in acute kidney Injury. PLoS One 2015;10:e0145042.
8. Clerico A, Galli C, Fortunato A, et al. Neutrophil gelatinaseassociated lipocalin (NGAL) as biomarker of acute kidney injury: a review of the laboratory characteristics and clinical evidences. Clin Chem Lab Med 2012;5017:1505.

9. Devarajan P. Neutrophil gelatinase-associated lipocalin: a promising biomarker for human acute kidney injury. Biomark Med 2010:4:265-80.

10. Nickolas TL, O'Rourke MJ, Yang J, et al. Sensitivity and specificity of a single emergency department measurement of urinary neutrophil gelatinase-associated lipocalin for diagnosing acute kidney injury. Ann Intern Med 2008;148:810-9.

11. Macdonald S, Arendts G, Nagree Y, et al. Neutrophil GelatinaseAssociated Lipocalin (NGAL) predicts renal injury in acute decompensated cardiac failure: a prospective observational study. BMC Cardiovasc Disord 2012;12:8.

12. Zhang A, Cai Y, Wang PF, et al. Diagnosis and prognosis of neutrophil gelatinase-associated lipocalin for acute kidney injury with sepsis: a systematic review and meta-analysis. Crit Care 2016;20:41.

13. Marino R, Struck J, Hartmann O, et al. Diagnostic and short-term prognostic utility of plasma pro-enkephalin (pro-ENK) for acute kidney injury in patients admitted with sepsis in the emergency department. J Nephrol 2015;28:717-24.

14. Akrawinthawong $\mathrm{K}$, Shaw MK, Kachner J, et al. Urine catalytic iron and neutrophil gelatinase-associated lipocalin as companion early markers of acute kidney injury after cardiac surgery: a prospective pilot study. Cardiorenal Med 2013;3:7-16.

15. Haase-Fielitz A, Haase M, Devarajan P. Neutrophil gelatinaseassociated lipocalin as a biomarker of acute kidney injury: a critical evaluation of current status. Ann Clin Biochem 2014;51:335-51.

16. Filiopoulos V, Biblaki D, Vlassopoulos D. Neutrophil GelatinaseAssociated Lipocalin (NGAL): a promising biomarker of contrastinduced nephropathy after computed tomography. Ren Fail 2014;36:979-86.

17. Haase M, Bellomo R, Devarajan P, et al. Accuracy of Neutrophil Gelatinase-Associated Lipocalin (NGAL) in diagnosis and prognosis in acute kidney injury: a systematic review and meta-analysis. Am J Kidney Dis 2009;54:1012-24.

18. Kashani K, Al-Khafaji A, Ardiles T, et al. Discovery and validation of cell cycle arrest biomarkers in human acute kidney injury. Crit Care 2013;17:R25.

19. Bihorac A, Chawla LS, Shaw AD, et al. Validation of cell-cycle arrest biomarkers for acute kidney injury using clinical adjudication. Am J Respir Crit Care Med 2014;189:932-9.

20. McCullough PA, Bouchard J, Waikar SS, et al. Implementation of novel biomarkers in the diagnosis, prognosis, and management of acute kidney injury: executive summary from the tenth consensus conference of the Acute Dialysis Quality Initiative (ADQI). Contrib Nephrol 2013;182:5-12. 